\title{
Changes in microRNA expression in the brachial plexus avulsion model of neuropathic pain
}

\author{
YUZHOU LIU ${ }^{1}, \mathrm{LE} \mathrm{WANG}^{1}, \mathrm{JIE} \mathrm{LAO}^{1-3}$ and XIN ZHAO ${ }^{1}$ \\ ${ }^{1}$ Department of Hand Surgery, Huashan Hospital, Fudan University; ${ }^{2}$ Key Laboratory of Hand Reconstruction, \\ Ministry of Health; ${ }^{3}$ Shanghai Key Laboratory of Peripheral Nerve and Microsurgery, Shanghai 200040, P.R. China
}

Received July 18, 2016; Accepted November 27, 2017

DOI: 10.3892/ijmm.2017.3333

\begin{abstract}
The present study aimed to perform microRNA (miRNA/miR) expression profiling of the thalamus (T), the anterior cingulate (AC), the dorsal horn of the spinal cord (DHSC) and the blood (B) in post-complete brachial plexus avulsion (CBPA) pain model, and analyze biological functions. Neuropathic pain was induced in Sprague-Dawley rats by CBPA. Animal behavioral tests were performed to differentiate the pain and control groups. DHSC, T, AC and B tissues were collected from the two groups for miRNA array analysis. The predicted mRNA targets were investigated by Gene Ontology analysis and pathway analysis. The results revealed that in the post-CBPA pain model, there were 10 differentially expressed miRNAs revealed among 4 different tissues. A total of 4 microRNAs in the AC and 3 microRNAs in the $\mathrm{T}$ were shown to be significantly upregulated. The functions of the differentially expressed miRNAs in the $\mathrm{AC}$ and $\mathrm{T}$ were synergetic in the aspect of positive regulation of neuron apoptotic process, inhibition of long-term potentiation and formation of synapse plasticity. miR-30c-1-3p and its predicted genes [calcium/calmodulin dependent protein kinase II $\beta$ (Camk2b) and protein kinase $\mathrm{C} \gamma$ (Prkcg)] existed in the $\mathrm{AC}$ and $\mathrm{T}$ groups with significant changes in expression. There were 2 miRNAs in the DHSC and B groups, respectively, with significant downregulation. The function of the change in miRNAs in the DHSC group was opposite to that in the $\mathrm{AC}$ and $\mathrm{T}$ groups. The differentially expressed microRNAs in the B group were revealed to be negative for the regulation of cell apoptosis. In conclusion, the central nerve groups (AC and $\mathrm{T}$ ) and the peripheral nerve group (DHSC) exhibited contrasting effects on synapse plasticity and neuron apoptosis. miR-30c-1-3p and its predicted genes (Camk2b and
\end{abstract}

Correspondence to: Dr Jie Lao, Department of Hand Surgery, Huashan Hospital, Fudan University, 12 Wulumuqi Zhong Road, Jing An, Shanghai 200040, P.R. China

E-mail: 1yz41_2000@sina.com

Key words: microRNA, brachial plexus, neuropathic pain, synapse plasticity, apoptosis
Prkcg) existed in the AC and T groups with significant changes in expression.

\section{Introduction}

Neuropathic pain caused by nerve injury remains an intractable disease due to a lack of satisfactory treatment (1-3). In 2009, Ciaramitaro et al (4) carried out a multicenter prospective study on the prevalence of neuropathic pain after traumatic brachial plexus injury. From the 107 patients enrolled, 56\% had neuropathic pain. Neuropathic pain impaired the quality of life and caused depression. Brachial plexus avulsion (BPA) induces a characteristic persistent oppression and intermittent shooting pain, which is often difficult to cure $(5,6)$. The pain could be experienced as burning or a feeling of compression. Pain after BPA is resistant to most traditional pain relief treatments (7) due to a lack of understanding of the cellular or molecular mechanisms in the occurrence and development of pain $(8,9)$.

As long-term sensitivity changes in neuropathic pain are associated with changes in gene regulation, it is worthy of note to determine if the microRNAs (miRNAs/miRs) that regulate genes taking part in the nociceptive pathways affect the occurrence and development of pain (10). The key roles of miRNAs in nervous system development and pathophysiology is increasingly evident (11), however, certain facts remain to be elucidated. Understanding the gene regulatory events in miRNA-mediated neuropathic pain may provide a pathway for identifying biomarkers or finding novel therapeutic targets (12). Since pathophysiological changes in pain are associated with altered expression in pain-associated proteins, miRNA may be a promising tool for controlling inflammatory and neuropathic pain by regulating gene and protein expression in pain pathways (13). Few studies have been performed on pain following brachial plexus injury (14-16).

In the present study, miRNA expression profiling was performed of the thalamus (T), the anterior cingulate (AC), the dorsal horn of the spinal cord (DHSC) and the blood (B) in a neuropathic pain model 4 weeks after complete brachial plexus avulsion (BPA) surgery. The combination of experimental and bioinformatics methods was applied to identify the biological and cytological functions that were influenced by the changes in miRNA expression (17). These data provide further evidence in support of the hypothesis that post-complete BPA pain may be regulated by miRNA. 


\section{Materials and methods}

Study approval. Animal handling and procedures used in this study were in agreement with the guidelines of the Animal Care and Use Committee of Fudan University (Shanghai, China). Neuropathic pain was induced in male Sprague-Dawley rats ( $n=40$; weight, 200-250 g; age, 8 weeks; supplied by the Department of Laboratory Animal Science, Fudan University, Shanghai, China) by CBPA. The rats were kept in an environment with a temperature of $20^{\circ} \mathrm{C}$ and humidity of $50 \%$ and were maintained on a 12/12-h light/dark cycle and allowed free access to food and water. The animal use protocol was reviewed and approved by the Animal Ethics and Welfare Committee of Huashan Hospital affiliated to Fudan University.

\section{Animal behavioral tests}

Mechanical allodynia. The rats were placed on a metal mesh floor, covered by a transparent plastic box and raised $30 \mathrm{~cm}$ above the floor. The plantar surface of the paw was stimulated with a series of ascending force von Frey monofilaments. The threshold was taken as the lowest force that evoked a brisk withdrawal response to one of five repetitive stimuli (7). A withdrawal response was considered valid only when the paw was completely removed from the platform.

Cold allodynia. Cold allodynia was measured by an acetone spray test, as described by Choi et al (18). Briefly, $250 \mathrm{ml}$ acetone was squirted onto the mid-plantar surface of the paw. The withdrawal responses were evaluated on a scale of $0-3$ points: 0 points, the paw was not moved; 1 point, a response in which the paw had little or no weight born on it; 2 points, a response in which the paw was elevated and was not in contact with any surface; and 3 points, a vigorous response in which the rat licked, bit or shook the paw.

All behavioral tests were performed by the same technician who was blinded to the study groups and identification of animals in order to avoid subjective differences in interpretation, which could occur with different observers (19).

Surgery procedure. Following the animal behavioral tests, the rats were anesthetized with sodium pentobarbital injected intraperitoneally. The CBPA rat model (20) was used to induce neuropathic pain. The brachial plexus was approached through a horizontal supraclavicular incision. The sternocleidomastoid muscle was cut off and the omohyoid muscle was pulled aside, leaving the transverse cervical vessels intact. The brachial plexus was located in the scalene fissure, including upper, middle and lower trunks. The complete brachial plexus was grasped with forceps and extracted from the spinal cord by traction. The tissue layers were then brought together and the skin was closed with 4-0 silk sutures.

These rats were maintained on a 12/12 h light/dark cycle and allowed free access to food and water for one month. At the end of the 4 weeks, paw withdrawal threshold to mechanical stimuli was assessed using von Frey filaments and cold allodynia was measured by an acetone spray test, as previously described.

According to the comparison of the behavioral test results (both mechanical allodynia and cold allodynia tests) prior to and following surgery, the rats were divided into two groups: The pain and control group. In the pain group, the paw withdrawal thresholds decreased and cold allodynia points rose following surgery, while the two results did not significantly change following surgery in the control group.

At the completion of behavioral testing, each group $(n=3)$ was euthanized using $\mathrm{CO}_{2}$. The flow rate for $\mathrm{CO}_{2}$ euthanasia displaced $20 \%$ of the chamber volume/min according to the 2013 edition of the American Veterinary Medical Association Guidelines for the Euthanasia of Animals. The following tissues were collected from these rats for microRNA analysis: DHSC, T, AC and B.

miRNA microarray. Total RNA was isolated using an miRNeasy mini kit and RNase-Free DNase set (both Qiagen, Inc., Valencia, CA, USA). RNA quality was checked with denaturing agarose gel (1.5\%) electrophoresis and nucleic acid staining. Samples with $28 \mathrm{~S}$ and $18 \mathrm{~S}$ rRNA bands were resolved into two discrete bands that had no significant smearing below each band, and the 28S rRNA band intensity, which was approximately twice that of the 18S rRNA band, was used for subsequent procedures. Total RNA $(1 \mu \mathrm{g})$ was labeled with Affymetrix ${ }^{\circledR}$ FlashTag $^{\mathrm{TM}}$, Biotin HSR RNA Labeling kits (Affymetrix, Inc., Santa Clara, CA, USA). Next, samples were hybridized to a GeneChip ${ }^{\circledR}$ miRNA 4.0 array (Affymetrix, Inc.) at $60 \mathrm{rpm}$, at $48^{\circ} \mathrm{C}$ for $16 \mathrm{~h}$. Fluorescent images of microarray slides were scanned using a GeneChip ${ }^{\circledR}$ Scanner 3000 7G (Affymetrix, Inc.). All CEL files from the miRNA 4.0 chips were normalized using Expression Console software (version 1.3.1; Affymetrix, Inc.). Affymetrix miRNA arrays are designed to contain all miRNAs in miRBase release 20 (http://tools.thermofisher.com/content/sfs/brochures/ miRNA_4-0_and_4-1_datasheet.pdf).

Reverse transcription-quantitative polymerase chain reaction (RT-qPCR) assay. All procedures were performed according to the corresponding manufacturer's protocols. Expression of the miRNAs was examined with the SYBR-Green RT-PCR kit (Invitrogen; Thermo Fisher Scientific, Inc., Waltham, MA, USA). Results were normalized to U6 expression. RT was performed with $1 \mathrm{mg}$ total RNA using the ThermoScript RT-PCR system (Invitrogen; Thermo Fisher Scientific. Inc.) for first-strand complementary DNA (cDNA) synthesis. For qPCR, cDNA was amplified using the MyiQ Real-Time PCR Detection system (Bio-Rad Laboratories, Inc., Hercules, CA, USA). Quantitative cycle values were obtained from each amplification curve using iQ5 Optical System Software (version 2.1) provided by the manufacturer (Bio-Rad Laboratories, Inc.). The $2^{-\Delta \Delta c t}$ method was used (21). Primer sequences will be provided upon request. The primers used were as follows: rno-miR-3573-5p forward, 5'-ACACTC CAGCTGGGTGAGGGGCAGTGATAGAAAGGA-3' and reverese, 5'-CTCAACTGGTGTCGTGGAGTCGGCAATTC AGTTGAGTCCTTTC-3'; rno-miR-3074 forward, 5'-ACACT CCAGCTGGGGATATCAGCTCAGTAGGCACCG-3' and reverse, CTCAACTGGTGTCGTGGAGTCGGCAATTCAG TTGAGCGGTGCC-3'; rno-miR-25-5p forward, 5'-ACACTCC AGCTGGGAGGCGGAGACACGGGCAATTGC-3' and reverse, 5'-CTCAACTGGTGTCGTGGAGTCGGCAATTCA GTTGAGGCAATTGC-3'; rno-miR-702-3p forward, 5'-ACA CTCCAGCTGGGTGCCCACCCTTTACCCCACTCCA-3' and reverse, 5'-CTCAACTGGTGTCGTGGAGTCGGCAATTCA GTTGAGTGGAGTG-3'; rno-miR-30c-1-3p forward, 5'-ACA CTCCAGCTGGGCTGGGAGAGGGTTGTTTACTCC-3' and 
reverse, 5'-CTCAACTGGTGTCGTGGAGTCGGCAATTC AGTTGAGGGAGTAAA-3'; rno-miR-93-3p forward, 5'-ACA CTCCAGCTGGGACTGCTGAGCTAGCACTTCCCGA-3' and reverse, 5'-CTCAACTGGTGTCGTGGAGTCGGCAATTCA GTTGAGTCGGGAAG-3'; rno-miR-873-5p forward, 5'-ACA CTCCAGCTGGGGCAGGAACTTGTGAGTCTCCT-3' and reverse, 5'-CTCAACTGGTGTCGTGGAGTCGGCAATTCA GTTGAGAGGAGAC-3'; rno-miR-455-3p forward, 5'-ACAC TCCAGCTGGGGCAGTCCACGGGCATATA-3' and reverse, 5'-CTCAACTGGTGTCGTGGAGTCGGCAATTCAGTTGA GAGTGTATA-3'; rno-miR-32-3p forward, 5'-ACACTCCAG CTGGGTGGACGGAGAACTGAT-3' and reverse, 5'-CTCA ACTGGTGTCGTGGAGTCGGCAATTCAGTTGAGACCC TTAT-3'; rno-miR-184 forward, 5'-ACACTCCAGCTGGGGC AATTTAGTGTGTGTGA-3' and reverse, 5'-CTCAACTGG TGTCGTGGAGTCGGCAATTCAGTTGAGAATATCAC-3'; universal miR reverse, 5'-TGTCGTGGAGTCGGCAATTC-3'; U6 forward, 5'-CTCGCTTCGGCAGCACATA-3' and reverse, 5'-ATATGGAACGCTTCACGAATTTGC-3'. The thermocycling conditions were as follows: stage 1 (1 cycle), pre-degeneration at $95^{\circ} \mathrm{C}$ for $5 \mathrm{sec}$; stage 2 (40 cycles), denaturation at $95^{\circ} \mathrm{C}$ for $5 \mathrm{sec}$ and primer annealing at $61^{\circ} \mathrm{C}$ for $30 \mathrm{sec}$; stage 3 ( 1 cycle), sufficient extention at $72^{\circ} \mathrm{C}$ for $1 \mathrm{~min}$ and cooling down at $4-10^{\circ} \mathrm{C}$.

Bioinformatic evaluation. For the differential regulation miRNAs, the predicted, but not (yet) verified mRNA targets were investigated. Since hundreds were found for each, Gene Ontology (GO) analysis (http://www.geneontology.org/page/go-database) wasperformedusingtheGOenrichmentanalysissoftwaretoolkit(22,23) and pathway analysis was performed using Kyoto Encyclopedia of Genes and Genomes (KEGG) pathway/brite/module mapping tools (24) (http://www.kegg.jp/kegg/pathway.html\#mapping) to define which gene molecular function terms were enriched that characterize the collection of target genes in comparison with the genome in general.

Statistical analysis. Due to the small sample size $(n=3)$, the random variance model t-test was adopted to filter the differentially expressed microRNAs between the control and pain groups using GraphPad 5.0. Following the significance analysis and false discovery rate analysis, differentially expressed genes were selected according to their P-values. $\mathrm{P}<0.05$ was considered to indicate a statistically significant difference.

\section{Results}

miRNA microarray. To functionally investigate a possible link between miRNA expression and the post-brachial plexus injury neuropathic pain, the differential expression of miRNAs between groups in 4 different tissues, DHSC, T, AC and B, was analyzed. The expression of $728 \mathrm{miRNAs}$ was detected in each tissue and divided into three types according to the changes: Decrease, increase and no change. The statistical results of the microRNA array analysis of the 4 different tissues are shown in Table I $(\mathrm{P}<0.05 ; \mathrm{n}=3)$. The statistical analysis software used was GraphPad 5.0.

In the DHSC group, 332 miRNAs exhibited decreased expression, 378 miRNAs exhibited increased expression and 18 miRNAs exhibited no change. The statistical analysis showed that 2 miRNAs were downregulated and 3 miRNAs were upregulated significantly.

In the T group, 305 miRNAs exhibited decreased expression, 409 miRNAs exhibited increased expression and 14 miRNAs exhibited no change. The statistical analysis showed that 13 miRNAs were downregulated and 9 miRNAs were upregulated significantly.

In the AC group, 348 miRNAs exhibited decreased expression, 359 miRNAs exhibited increased expression and 21 miRNAs exhibited no change. The statistical analysis showed that 3 miRNAs were downregulated and 9 miRNAs were upregulated significantly.

In the B group, 362 miRNAs exhibited decreased expression, 352 miRNAs exhibited increased expression and 14 miRNAs exhibited no change. The statistical analysis showed that 7 miRNAs were downregulated and no miRNAs were upregulated significantly.

PCR verification. To validate the microarray results, $\mathrm{RT}-\mathrm{qPCR}$ was performed for these differentially regulated (diff-reg) miRNAs in the 4 groups. It was found that the relative expression of 10 miRNAs among them were significantly altered, which coincided with the results of the microarray. The 10 miRNAs were miR-3573-5p (DHSC), miR-3074 (DHSC), miR-30c-1-3p (AC and T), miR-702-3p (AC), miR-184 (AC), miR-25-5p (AC), miR-873-5p (T), miR-93-3p (T), miR-455-3p (B) and miR-32-3p (B). miR-30c-1-3p with a significant change appeared in both the AC and T groups. The other miRNAs could not be verified by PCR. The statistical analysis showed that miR-3573-5p (DHSC), miR-3074 (DHSC), miR-455-3p (B) and miR-32-3p (B) were downregulated, and that miR-30c-1-3p (AC and T), miR-702-3p (AC), miR-184 (AC), miR-25-5p (AC), miR-873-5p (T) and miR-93-3p (T) were upregulated significantly (Table II).

Bioinformatics analysis of the diff-reg miRNAs. For these diff-reg miRNAs with PCR verification, the predicted, but not (yet) verified, mRNA targets were investigated.

In the DHSC group, there were 27 intersection genes (Table III), which were involved in neuropathic pain according to GO and pathway analyses. GO analysis showed that the downregulation function of miR-3573-5p and miR-3074 associated with neuropathic pain included 'axon guidance', 'synaptic transmission', 'synapse maturation', 'excitatory post-synaptic membrane potential', 'neuron apoptotic process', 'macrophage activation involved in immune response', 'cell chemotaxis', 'neuron migration' and 'neuron differentiation'. The target genes of miR-3573-5p and miR-3074 took part in several pathways, including the 'calcium signaling pathway', 'cholinergic synapse', 'GABAergic synapse', 'glutamatergic synapse', the 'HIF-1 signaling pathway', the 'MAPK signaling pathway', the 'mTOR signaling pathway', the 'notch signaling pathway', the 'PI3K-Akt signaling pathway', 'synaptic vesicle cycle' and the 'Wnt signaling pathway'. Downregulation of miR-3573-5p and miR-3074 would result in upregulation of the target genes, which inhibited neuron apoptotic process, promoted long-term potentiation and synapse plasticity, and strengthened cell proliferation and differentiation.

In the $\mathrm{T}$ group, there were 3 intersection genes, which were involved in neuropathic pain according to GO and pathway 
Table I. miRNAs with statistical differences in expression between the pain and control groups.

\begin{tabular}{|c|c|c|c|c|c|c|}
\hline miRNA & P-value & FDR & $\begin{array}{l}\text { Fold- } \\
\text { change }\end{array}$ & $\begin{array}{l}\text { Regulation } \\
\text { trend }\end{array}$ & $\begin{array}{l}\text { Sequence } \\
\text { length, nt }\end{array}$ & Sequence $\left(5^{\prime}-3^{\prime}\right)$ \\
\hline \multicolumn{7}{|l|}{ DHSC group } \\
\hline miR-3573-5p & 0.0068 & 1.000 & 0.70 & Down & 22 & UGAGGGGCAGUGAUAGAAAGGA \\
\hline miR-3074 & 0.041413 & 1.000 & 0.90 & Down & 22 & GAUAUCAGCUCAGUAGGCACCG \\
\hline $\operatorname{miR}-1193-3 p$ & 0.025148 & 1.000 & 1.23 & Up & 22 & UAGGUCACCCGUUUUACUAUCC \\
\hline $\operatorname{miR}-410-5 p$ & 0.040567 & 1.000 & 1.23 & Up & 21 & AGGUUGUCUGUGAUGAGUUCG \\
\hline miR-340-5p & 0.018692 & 1.000 & 1.48 & Up & 22 & UUAUAAAGCAAUGAGACUGAUU \\
\hline \multicolumn{7}{|l|}{ AC group } \\
\hline $\operatorname{miR}-208 a-3 p$ & 0.018404 & 0.997 & 0.37 & Down & 18 & AUAAGACGAGCAAAAAGC \\
\hline $\operatorname{miR}-6216$ & 0.028229 & 0.997 & 0.71 & Down & 23 & GAUACACAGAGGCAGGAGGAGAA \\
\hline $\operatorname{miR}-3580-3 p$ & 0.034532 & 0.997 & 0.72 & Down & 22 & UGACUAGGGUAGUAUGAGUAGA \\
\hline miR-205 & 0.045209 & 0.997 & 1.41 & Up & 23 & UCCUUCAUUCCACCGGAGUCUGU \\
\hline miR-25-5p & 0.030307 & 0.997 & 1.46 & Up & 22 & AGGCGGAGACACGGGCAAUUGC \\
\hline miR-702-3p & 0.035705 & 0.997 & 1.49 & Up & 23 & UGCCCACCCUUUACCCCACUCCA \\
\hline miR-501-3p & 0.024475 & 0.997 & 1.65 & Up & 23 & AAUGCACCCGGGCAAGGAUUUGG \\
\hline let-7f-5p & 0.035378 & 0.997 & 1.70 & Up & 22 & UGAGGUAGUAGAUUGUAUAGUU \\
\hline $\operatorname{miR}-381-5 p$ & 0.032536 & 0.997 & 1.80 & Up & 22 & AGCGAGGUUGCCCUUUGUAUAU \\
\hline $\operatorname{miR}-30 c-1-3 p$ & 0.011214 & 0.997 & 1.87 & Up & 22 & CUGGGAGAGGGUUGUUUACUCC \\
\hline $\operatorname{miR}-671$ & 0.028852 & 0.997 & 1.87 & Up & 21 & UCCGGUUCUCAGGGCUCCACC \\
\hline $\operatorname{miR}-184$ & 0.006879 & 0.997 & 2.42 & Up & 22 & UGGACGGAGAACUGAUAAGGGU \\
\hline \multicolumn{7}{|l|}{$\mathrm{T}$ group } \\
\hline miR-34b-5p & 0.04851 & 0.971 & 0.62 & Down & 23 & AGGCAGUGUAAUUAGCUGAUUGU \\
\hline $\operatorname{miR}-181 d-3 p$ & 0.0342 & 0.971 & 0.65 & Down & 20 & CCACCGGGGGAUGAAUGUCA \\
\hline $\operatorname{miR}-484$ & 0.027441 & 0.971 & 0.66 & Down & 22 & UCAGGCUCAGUCCCCUCCCGAU \\
\hline miR-370-5p & 0.016922 & 0.971 & 0.70 & Down & 24 & CAGGUCACGUCUCUGCAGUUACAC \\
\hline $\operatorname{miR}-9 b-5 p$ & 0.008269 & 0.971 & 0.78 & Down & 19 & UUCGGUUAUCUAGCUUUAU \\
\hline $\operatorname{miR}-1912-3 p$ & 0.017277 & 0.971 & 0.79 & Down & 19 & CACAGAACAUGCAGUGAGA \\
\hline $\operatorname{miR}-759$ & 0.018461 & 0.971 & 0.81 & Down & 22 & GCAGAGUGCAAACAAUUUUGAC \\
\hline $\operatorname{miR}-463-5 p$ & 0.036635 & 0.971 & 0.81 & Down & 21 & UACCUAAUUUGUUGUCCAUCA \\
\hline miR-193-3p & 0.045474 & 0.971 & 0.81 & Down & 22 & AACUGGCCUACAAAGUCCCAGU \\
\hline $\operatorname{miR}-802-5 p$ & 0.040079 & 0.971 & 0.83 & Down & 21 & UCAGUAACAAAGAUUCAUCCU \\
\hline $\operatorname{miR}-218 a-5 p$ & 0.019683 & 0.971 & 0.84 & Down & 21 & UUGUGCUUGAUCUAACCAUGU \\
\hline $\operatorname{miR}-31 b$ & 0.031174 & 0.971 & 0.84 & Down & 19 & CUAUGCCAGCAUCUUGCCU \\
\hline $\operatorname{miR}-3593-5 p$ & 0.033797 & 0.971 & 0.87 & Down & 22 & UGGCCUCCGCAGGGUUGAAGCU \\
\hline $\operatorname{miR}-3570$ & 0.039038 & 0.971 & 1.15 & Up & 22 & GGUACAAUCAACGGUCGAUGGU \\
\hline $\operatorname{miR}-3588$ & 0.018922 & 0.971 & 1.19 & Up & 22 & UCACAAGUUAGGGUCUCAGGGA \\
\hline miR-664-3p & 0.039461 & 0.971 & 1.30 & Up & 22 & UAUUCAUUUACUCCCCAGCCUA \\
\hline $\operatorname{miR}-488-3 p$ & 0.025485 & 0.971 & 1.41 & Up & 21 & UUGAAAGGCUGUUUCUUGGUC \\
\hline $\operatorname{miR}-30 c-1-3 p$ & 0.009129 & 0.971 & 2.03 & Up & 22 & CUGGGAGAGGGUUGUUUACUCC \\
\hline $\operatorname{miR}-106 b-3 p$ & 0.018801 & 0.971 & 2.08 & Up & 22 & CCGCACUGUGGGUACUUGCUGC \\
\hline $\operatorname{miR}-93-3 p$ & 0.012217 & 0.971 & 2.69 & Up & 23 & ACUGCUGAGCUAGCACUUCCCGA \\
\hline $\operatorname{miR}-28-5 p$ & 0.041407 & 0.971 & 2.85 & Up & 22 & AAGGAGCUCACAGUCUAUUGAG \\
\hline $\operatorname{miR}-873-5 p$ & 0.02098 & 0.971 & 2.93 & Up & 21 & GCAGGAACUUGUGAGUCUCCU \\
\hline \multicolumn{7}{|l|}{ B group } \\
\hline $\operatorname{miR}-455-3 p$ & 0.028562 & 0.997 & 0.32 & Down & 22 & GCAGUCCACGGGCAUAUACACU \\
\hline $\operatorname{miR}-32-3 p$ & 0.030898 & 0.997 & 0.47 & Down & 22 & GCAAUUUAGUGUGUGUGAUAUU \\
\hline $\operatorname{miR}-466 b-2-3 p$ & 0.032782 & 0.997 & 0.57 & Down & 21 & AUAUACAUACACACAUACACA \\
\hline $\operatorname{miR}-702-3 p$ & 0.035054 & 0.997 & 0.62 & Down & 23 & UGCCCACCCUUUACCCCACUCCA \\
\hline miR-742-5p & 0.035279 & 0.997 & 0.72 & Down & 21 & UACUCACAUGGUUGCUAAUCA \\
\hline miR-195-3p & 0.021132 & 0.997 & 0.73 & Down & 23 & CCAAUAUUGGCUGUGCUGCUCCA \\
\hline miR-509-3p & 0.045567 & 0.997 & 0.77 & Down & 22 & UGAUUGACAUGUCUGCAGUGGA \\
\hline
\end{tabular}

miRNA/miR, microRNA; FDR, false discovery rate; DHSC, dorsal horn of the spinal cord; AC, anterior cingulate; T, thalamus; B, blood. 
Table II. miRNAs with differential expression by PCR verification between pain and control groups.

\begin{tabular}{lcccccc}
\hline miRNA & P-value & FDR & $\begin{array}{c}\text { Fold- } \\
\text { change }\end{array}$ & $\begin{array}{c}\text { Regulation } \\
\text { trend }\end{array}$ & $\begin{array}{c}\text { Sequence } \\
\text { length, nt }\end{array}$ & Sequence (5'-3') \\
\hline $\begin{array}{l}\text { DHSC group } \\
\text { rno-miR-3573-5p }\end{array}$ & 0.0067996 & 1.000 & 0.70 & Down & 22 & UGAGGGGCAGUGAUAGAAAGGA \\
rno-miR-3074 & 0.0414127 & 1.000 & 0.90 & Down & 22 & GAUAUCAGCUCAGUAGGCACCG \\
$\begin{array}{l}\text { AC group } \\
\text { rno-miR-25-5p }\end{array}$ & 0.0303073 & 0.997 & 1.46 & $\mathrm{Up}$ & 22 & AGGCGGAGACACGGGCAAUUGC \\
rno-miR-702-3p & 0.0357045 & 0.997 & 1.49 & $\mathrm{Up}$ & 23 & UGCCCACCCUUUACCCACUCCA \\
rno-miR-30c-1-3p & 0.0112141 & 0.997 & 1.87 & $\mathrm{Up}$ & 22 & CUGGGAGAGGGUUGUUUCUCC \\
rno-miR-184 & 0.0068785 & 0.997 & 2.42 & $\mathrm{Up}$ & 22 & UGGACGGAGAACUGAUAAGGGU \\
$\begin{array}{l}\text { T group } \\
\text { rno-miR-30c-1-3p }\end{array}$ & 0.0091288 & 0.971 & 2.03 & $\mathrm{Up}$ & 22 & CUGGGAGAGGGUUGUUUACUCC \\
rno-miR-93-3p & 0.0122168 & 0.971 & 2.69 & $\mathrm{Up}$ & 23 & ACUGCUGAGCUAGCACUUCCCGA \\
rno-miR-873-5p & 0.02098 & 0.971 & 2.93 & $\mathrm{Up}$ & 21 & GCAGGAACUUGUGAGUCUCCU \\
$\begin{array}{l}\text { B group } \\
\text { rno-miR-455-3p }\end{array}$ & 0.0285622 & 0.997 & 0.32 & Down & 22 & GCAGUCCACGGGCAUAUACACU \\
rno-miR-32-3p & 0.0308978 & 0.997 & 0.47 & Down & 22 & GCAAUUUAGUGUGUGUGAUAUU \\
\hline
\end{tabular}

miRNA/miR, microRNA; FDR, false discovery rate; DHSC, dorsal horn of the spinal cord; AC, anterior cingulate; T, thalamus; B, blood.

Table III. Intersection genes involved in neuropathic pain in GO and pathway analyses.

\begin{tabular}{|c|c|}
\hline Group & Genes \\
\hline DHSC & $\begin{array}{l}\text { Grin1, Grm4, Grm6, Itga5, Notch1, P2rx1, Prkcg, } \\
\text { Shank1, Peg12, Shank3, Srf, Stx 1b, Stxbp1, Syk, } \\
\text { Vegfa, Vhl, Wnt8b, Wif1, Adra1b, Cacna1a, Cd38, } \\
\text { Chat, Ddit4, Efna2, Fgfr3, Gad1, Gng11 }\end{array}$ \\
\hline $\mathrm{AC}$ & $\begin{array}{l}\text { Camk2b, Csf1, Hdac1, Kdr, Mapk3, Prkcg, Tgfa, } \\
\text { Vegfa }\end{array}$ \\
\hline $\mathrm{T}$ & Camk2b, Itgb1, Prkcg \\
\hline B & Scn1a, Kdr, Nos3 \\
\hline
\end{tabular}

Genes listed belong to the targets of differentially expressed microRNAs according to GO and pathway analyses. GO, Gene Ontology; DHSC, dorsal horn of the spinal cord; AC, anterior cingulate; T, thalamus; B, blood.

analyses (Table III). GO analysis showed that the upregulation function of miR-30c-1-3p, miR-873-5p and miR-93-3p associated with neuropathic pain included 'neurotransmitter uptake', 'neuron apoptotic process', 'sensory perception of pain', 'long-term memory' and 'neuron projection development'. The target genes of miR-30c-1-3p, miR-873-5p and miR-93-3p took part in several pathways, including the 'calcium signaling pathway', 'cholinergic synapse', 'dopaminergic synapse', the 'ErbB signaling pathway', 'GABAergic synapse', 'glutamatergic synapse', the 'hedgehog signaling pathway', the 'HIF-1 signaling pathway', 'long-term potentiation', the 'MAPK signaling pathway', the 'mTOR signaling pathway', the 'neurotrophin signaling pathway', the 'notch signaling pathway', the 'PI3K-Akt signaling pathway', 'retrograde endocannabinoid signaling', 'serotonergic synapse' and the 'Wnt signaling pathway'. Upregulation of these miRNAs would result in downregulation of the target genes, which promoted neuron apoptotic apoptosis, reduced long-term potentiation and synapse plasticity, and inhibited cell proliferation and differentiation.

In the $\mathrm{AC}$ group, there were 8 intersection genes, which were involved in neuropathic pain according to GO and pathway analyses (Table III). GO analysis indicated that the upregulation function of miR-30c-1-3p, miR-702-3p, miR-184 and miR-25-5p associated with neuropathic pain included 'neuron apoptotic process', 'regulation of neuronal synaptic plasticity', 'axon guidance', 'synaptic transmission (glutamatergic)', 'axon extension', 'axonogenesis', 'long-term synaptic potentiation', 'sensory perception of pain', 'response to cold', 'response to stress', 'neuron differentiation', 'neuron migration', 'neuron cell-cell adhesion' and 'long-term memory'. The target genes of miR-30c-1-3p, miR-702-3p, miR-184 and miR-25-5p took part in several pathways, including the "calcium signaling pathway', 'cholinergic synapse', the 'ErbB signaling pathway', 'GABAergic synapse', 'glutamatergic synapse', the 'hedgehog signaling pathway', the 'HIF-1 signaling pathway', 'long-term depression', the 'mTOR signaling pathway', the 'MAPK signaling pathway', 'dopaminergic synapse', 'long-term potentiation', 'serotonergic synapse', the 'neurotrophin signaling pathway', the 'notch signaling pathway', the 'PI3K-Akt signaling pathway', 'retrograde endocannabinoid signaling' and the 'Wnt signaling pathway'. Upregulation of these miRNAs would result in downregulation of the target genes, which promoted neuron apoptotic apoptosis, reduced long-term potentiation and synapse plasticity, and inhibited cell proliferation and differentiation. 


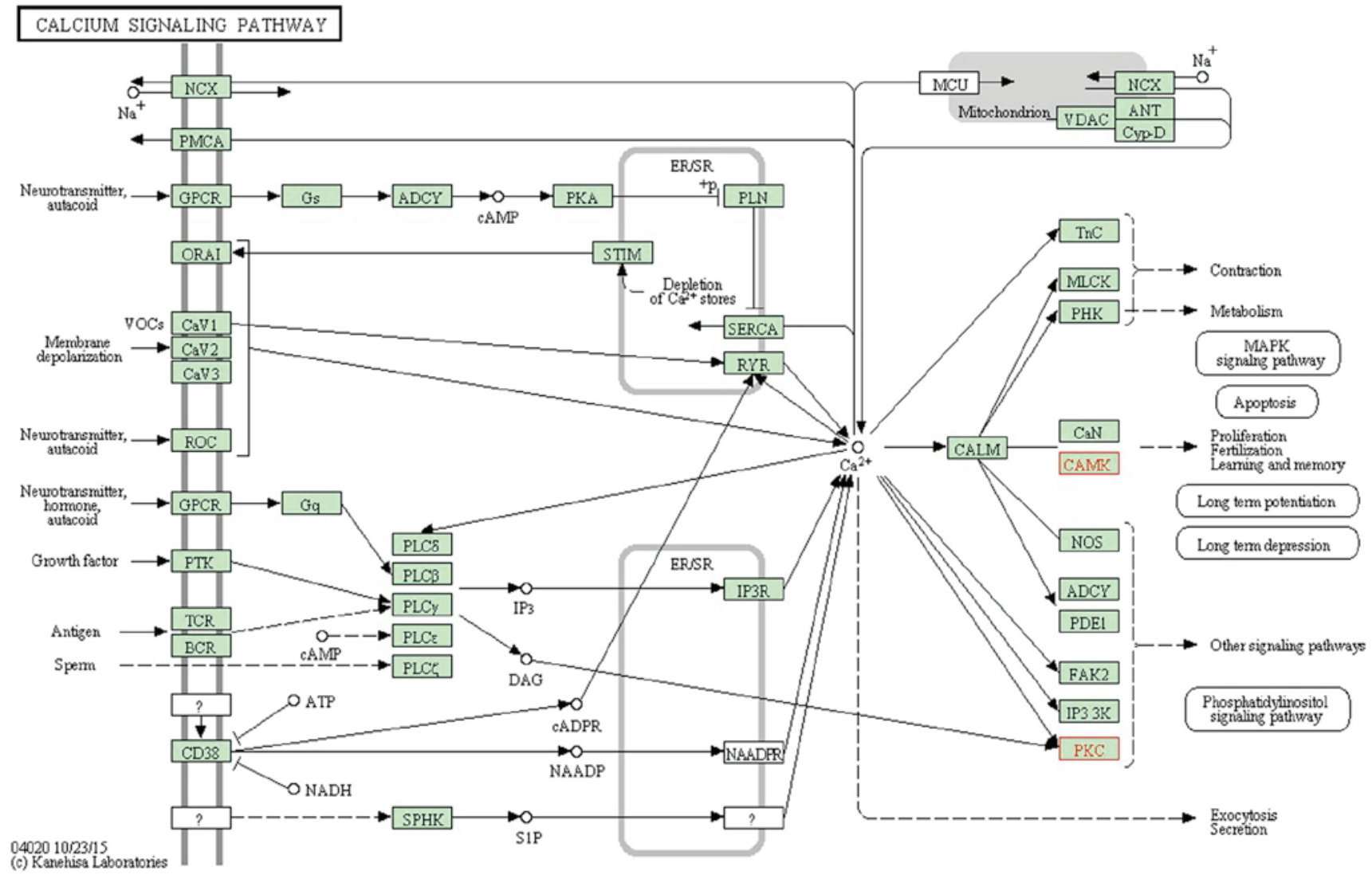

Figure 1. Calcium signaling pathway, with red font indicating target genes. The downregulation of Camk2b and Prkeg would inhibit cell proliferation. This figure was obtained using the KEGG PATHWAY mapping tool in the KEGG database (www.kegg.jp/dbget-bin/www_bget?map04020) and published with permission from Kanehisa Laboratories (23-25). KEGG, Kyoto Encyclopedia of Genes and Genomes.

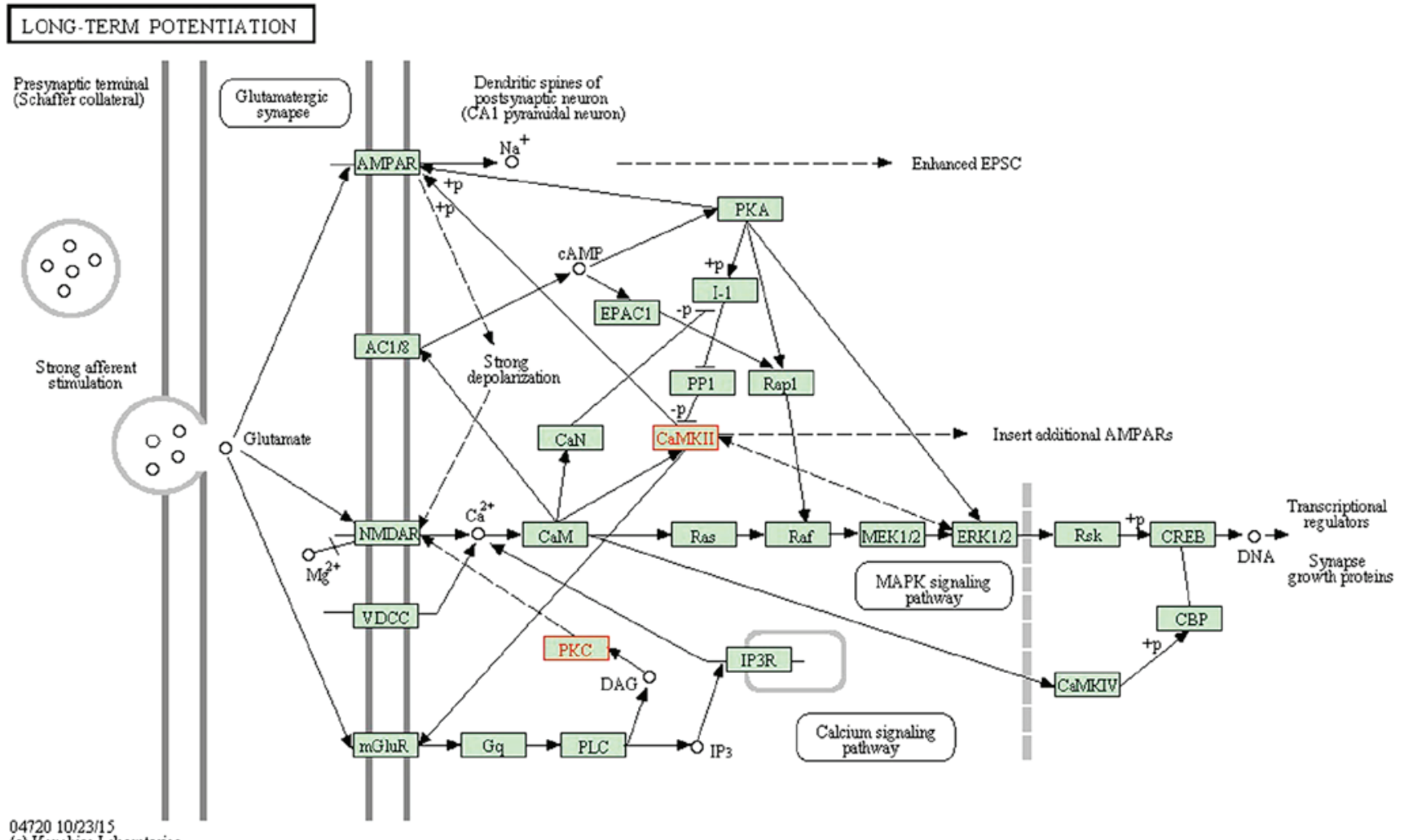

(c) Kanehisa Laboratonies

Figure 2. Long-term potentiation, with red font indicating target genes. The downregulation of Camk2b and Prkcg would inhibit synapse growth protein formation. This figure was obtained using the KEGG PATHWAY mapping tool in the KEGG database (www.kegg.jp/dbget-bin/www_bget?map04720) and published with permission from Kanehisa Laboratories (23-25). KEGG, Kyoto Encyclopedia of Genes and Genomes. 


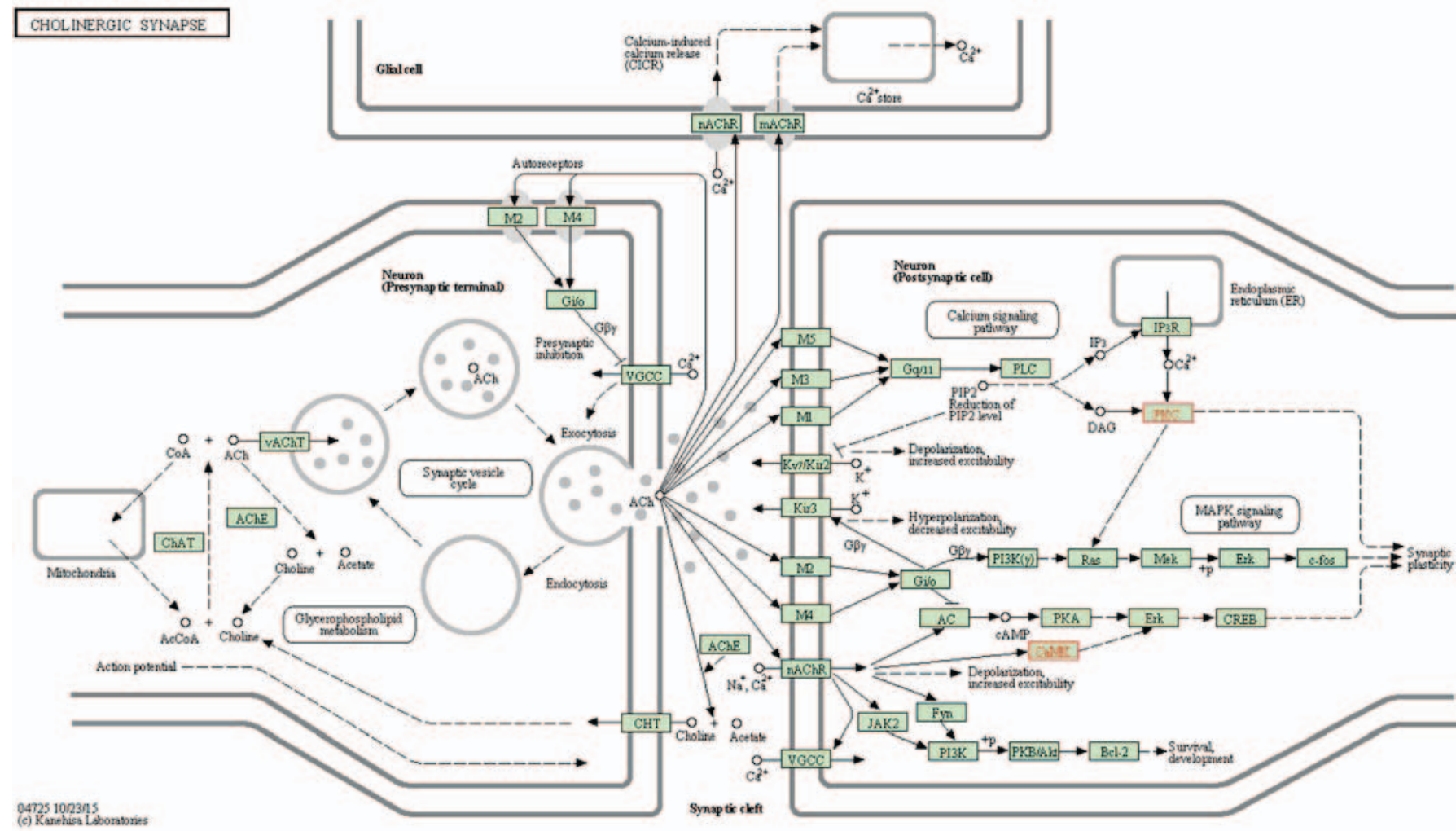

Figure 3. Cholinergic synapse, with red font indicating target genes. The downregulation of Camk2b and Prkcg would decrease synapse plasticity. This figure was obtained using the KEGG PATHWAY mapping tool in the KEGG database (www.kegg.jp/dbget-bin /www_bget?map04725) and published with permission from Kanehisa Laboratories (23-25). KEGG, Kyoto Encyclopedia of Genes and Genomes.

In the $\mathrm{B}$ group, there were 3 intersection genes, which were involved in neuropathic pain according to GO and pathway analyses (Table III). GO analysis indicated that the downregulation function of miR-455-3p and miR-32-3p associated with neuropathic pain included 'neuron projection morphogenesis', 'cell migration', 'cell proliferation' and 'apoptotic process'. The target genes of miR-455-3p and miR-32-3p took part in four pathways: The 'calcium signaling pathway', the 'HIF-1 signaling pathway', the 'PI3K-Akt signaling pathway' and the 'VEGF signaling pathway'. Downregulation of miR-455-3p and miR-32-3p would induce upregulation of the target genes, which inhibited cell apoptosis, and promoted vascularization, cell migration and proliferation.

In the post-brachial plexus injury neuropathic pain model, the central nerve groups (AC and T) and the peripheral nerve group (DHSC) exhibited contrasting effects on synapse plasticity and neuron apoptosis. The former showed downregulation of synapse plasticity, cell proliferation and differentiation, while the later showed upregulation.

miR-30c-1-3p with a significant change appeared in the AC and $\mathrm{T}$ groups, which significantly increased in the pain model. The functions of miR-30c-1-3p associated with neuropathic pain included the 'calcium signaling pathway', 'cholinergic synapse', 'dopaminergic synapse', the 'HIF-1 signaling pathway', the 'ErbB signaling pathway', 'long-term potentiation', the 'neurotrophin signaling pathway', the 'Wnt signaling pathway', 'GABAergic synapse', 'glutamatergic synapse', the 'MAPK signaling pathway', the 'mTOR signaling pathway', 'retrograde endocannabinoid signaling', 'serotonergic synapse' and 'long-term depression'. The predicted genes of miR-30c-1-3p were calcium/calmodulin dependent protein kinase II $\beta$ (Camk2b) and protein kinase $\mathrm{C} \gamma$ (Prkcg), which were validated by PCR to exhibit significant downregulation in the AC and T groups. Camk2b and Prkcg took part in the "calcium signaling pathway' (www.kegg.jp/dbget-bin/www_bget?map04020; Fig. 1), 'long-term potentiation' (www.kegg.jp/dbget-bin/ www_bget?map04720; Fig. 2) and 'cholinergic synapse' (www.kegg.jp/dbget-bin/www_bget?map04725; Fig. 3). All figures were obtained using the KEGG pathway mapping tool in the KEGG database (24-26). The downregulation of Camk2b and Prkcg would inhibit cell proliferation and synapse growth protein formation, which made synapse plasticity decrease.

\section{Discussion}

It has previously been revealed that descending facilitatory modulation is a key mechanism underlying the induction and maintenance of neuropathic pain (27). It is well documented that spinal nociception is powerfully modulated by an endogenous descending inhibitory system (27), although descending control is bi-directional via inhibitory and facilitatory systems. When the brachial plexus was avulsed, the peripheral nerves were separated from the DHSC, which resulted in blockage of the descending inhibitory system. Sustained activation of descending facilitation increased the excitability of spinal nociceptive neurons and promoted long-term potentiation and synapse plasticity, which in turn evoked neuroplasticity of certain supraspinal structures, including the anterior cingulate cortex (ACC) and rostral ventral medulla (8-30), which underly certain states of neuropathic pain. 
The roles of the ACC and T in pain conditions have been consistently demonstrated for the past several decades $(31,32)$. Numerous animal studies have been performed to identify the $\mathrm{T}$ and $\mathrm{ACC}$ as potential mediators of the pain experience, which are not only involved in the transmission of pain sensation, but also serve a role in processing pain-related emotions (27). The ACC and T widely connect with the descending modulation system.

However, the miRNAs within the ACC and T that mediated the perception of nociceptive signals following brachial plexus injury remain poorly understood. In the present study, 4 miRNAs in the ACC and 3 miRNAs in the T exhibited significant upregulation, and were associated with neuropathic pain. The functions of the miRNAs in the ACC and T were synergetic in the aspect of positive regulation of neuron apoptotic process, and inhibition of long-term potentiation and synapse plasticity. Previous studies reported that peripheral injury induced long-term potentiation of excitatory synaptic responses in the ACC neurons $(33,34)$.

miR-30c-1-3p and its predicted genes (Camk2b and Prkcg) existed in the $\mathrm{AC}$ and $\mathrm{T}$ groups, with significant changes, in the present study. Waggener et al (35) reported that calmodulindependent protein kinase II regulated oligodendrocyte maturation and central nervous system myelination. Fang et al (36) reported that Camk2b protected neurons from homocysteine-induced apoptosis with the involvement of the hypoxia-inducible factor $1 \alpha$ signal pathway. Miletic et al (37) demonstrated that Prkcg mediated the phosphorylation of glutamate receptor 1 in the postsynaptic density of spinal dorsal horn neurons, accompanied with neuropathic pain. Yeh et al (38) observed the expression of dorsal spinal protein kinase $\mathrm{C} \gamma$-subunit and other pain-related molecules in the spinal area in neuropathic pain model animals. Together, these studies indicated that miR-30c-1-3p may serve an extremely important role in neuropathic pain.

Besides central sensitization, peripheral sensitization is also associated with neuropathic pain following brachial plexus avulsion, which may result from ectopic discharge (39), scar stimulation (40) and excitory conduction short circuit due to the lack of myelin sheaths, among others (41). The concrete mechanisms of these causes include five aspects: Neuronal apoptosis, axonal regeneration, immune cell infiltration, glial cell accumulation and chemokine transfer (42). The present study indicated that 2 microRNAs in the DHSC group and 2 microRNAs in the B group exhibited significant downregulation in the pain model. GO analysis showed that these microRNAs had functions in 'axon guidance', 'synaptic transmission', 'synapse maturation', 'excitatory postsynaptic membrane potential', 'the neuronal apoptotic process', 'macrophage activation involved in the immune response', 'cell chemotaxis', 'neuron migration' and 'neuron differentiation', 'neuron projection morphogenesis', 'cell migration', 'cell proliferation' and 'apoptosis', which facilitated the peripheral sensitization and induced neuropathic pain. The functions of the microRNAs in the DHSC and B groups were synergetic in the aspect of inhibition of cell apoptosis and promotion of cell proliferation, which contrasted with the functions of the differentially expressed miRNAs in the $\mathrm{AC}$ and $\mathrm{T}$ groups. When sustained activation of descending facilitation system occurred, a number of neurotrophic factors and neurotransmitters were exhausted and synapse growth proteins decreased, which resulted in neuron apoptosis increasing and synapse plasticity decreasing in the $\mathrm{AC}$ and $\mathrm{T}$.

There were 10 miRNAs with significant expressional changes in the pain group, namely miR-3573-5p (DHSC), miR-3074(DHSC), miR-30c-1-3p (AC and T), miR-702-3p (AC), miR-184 (AC), miR-25-5p (AC), miR-873-5p (T), miR-93-3p (T), miR-455-3p (B) and miR-32-3p (B). Previous studies on the aforementioned miRNAs associated with neuropathic pain have included a study by Lu et al (43), which found that miR-702-3p was differentially expressed in rat cortex in the anesthetic treatment group, which indicated the miRNA may be associated with neuropathic pain. McAdams et al (44) reported that morphine decreased the expression of miR-455-3p in the hippocampus of stressed neonatal mice and that the miRNA was involved in neurodevelopment, neurotransmission and inflammation. Gong et al (45) investigated the differentially expressed miRNAs in the lumbar spinal dorsal horn of mice with streptozotocin-induced diabetic neuropathic pain (DNP) and found that aberrant expression of miR-184 may contribute to the pathogenesis of DNP, and was a potential target for therapeutic interventions following DNP. McKiernan et al (46) identified miR-184 as a novel contributor to neuronal survival following mild and severe seizures. Furthermore, Liu et al (47) found that high levels of miR-184 promoted proliferation, but inhibited differentiation of adult neural stem/progenitor cells, and that the miRNA regulated the expression of numblike, a known regulator of brain development.

In future research, the predicted genes $(\mathrm{Camk} 2 \mathrm{~b}$ and Prkcg) should be verified as the targets of miR-30c-1-3p by cytology. miR-30c-1-3p could be transfected into neuron cells through plasmids and then the amount of target proteins could be measured by PCR and western blot analysis. If Camk $2 b$ and Prkcg were validated as the targets of miR-30c-1-3p, an attempt would be made to inject an miR-30c-1-3p-expressing virus into the ACC of the rat and then behavioral tests would be performed to determine whether the rat was in pain or not.

In conclusion, in the present study, post-CBPA pain was regulated intricately by miRNAs from different tissues in different pathways. There were 10 miRNAs with significant changes in expression in 4 different tissues in the post-BPA pain model. The central nerve groups ( $\mathrm{AC}$ and $\mathrm{T})$ and the peripheral nerve groups (DHSC) exhibited contrasting effects on synapse plasticity and neuron apoptosis. miR-30c-1-3p and its predicted genes (Camk2b and Prkcg) existed in the AC and $\mathrm{T}$ groups with significant espressional changes.

\section{Acknowledgements}

The authors would like to thank Dr Jiali Li (Fudan University) for providing assistance with the data analysis. The present study was sponsored by the Ministry of Science and Technology of China (973 program; grant no. 2014CB542204), the Natural Science Foundation of Shanghai (grant no. STCSM 16ZR1404200) and the National Natural Science Foundation of China (grant no. NSFC 81572127).

\section{References}

1. Mendell JR and Sahenk Z: Clinical practice. Painful sensory neuropathy. N Engl J Med 348: 1243-1255, 2003. 
2. Sawynok J: Topical and peripherally acting analgesics. Pharmacol Rev 55: 1-20, 2003.

3. Hansson PT and Dickenson AH: Pharmacological treatment of peripheral neuropathic pain conditions based on shared commonalities despite multiple etiologies. Pain 113: 251-254, 2005.

4. Ciaramitaro P,Padua L, Devigili G, Rota E, Tamburin S, Eleopra R, Cruccu G and Truini A; Neuropathic pain special interest group of the Italian Neurological Society: Prevalence of neuropathic pain in patients with traumatic brachial plexus injury: A multicenter prospective hospital-based study. Pain Med: March 3,2017 (Epub ahead of print)

5. Anand P and Birch R: Restoration of sensory function and lack of long-term chronic pain syndromes after brachial plexus injury in human neonates. Brain 125: 113-122, 2002.

6. Berman JS, Birch R and Anand P: Pain following human brachial plexus injury with spinal cord root avulsion and the effect of surgery. Pain 75: 199-207, 1998.

7. Baruah S, Devi BI, Bhat DI and Shukla D: Drezotomy in the management of post brachial plexus injury neuropathic pain Preliminary results. Indian J Neurotrauma 11: 27-29, 2014

8. Aley KO and Levine JD: Different peripheral mechanisms mediate enhanced nociception in metabolic/toxic and traumatic painful peripheral neuropathies in the rat. Neuroscience 111: 389-397, 2002.

9. Erichsen HK and Blackburn-Munro G: Pharmacological characterisation of the spared nerve injury model of neuropathic pain Pain 98: 151-161,2002.

10. Aldrich BT, Frakes EP, Kasuya J, Hammond DL and Kitamoto T: Changes in expression of sensory organ-specific microRNAs in rat dorsal root ganglia in association with mechanical hypersensitivity induced by spinal nerve ligation. Neuroscience 164 711-723, 2009

11. Bhalala OG, Srikanth M and Kessler JA: The emerging roles of microRNAs in CNS injuries. Nat Rev Neurol 9: 328-339, 2013.

12. Erson AE and Petty EM: MicroRNAs in development and disease. Clin Genet 74: 296-306, 2008.

13. Tan PH, Pao YY, Cheng JK, Hung KC and Liu CC: MicroRNA-based therapy in pain medicine: Current progress and future prospects. Acta Anaesthesiol Taiwan 51: 171-176, 2013.

14. Parry CB: Pain in avulsion lesions of the brachial plexus. Pain 9 41-53, 1980.

15. Narakas AO: The effects on pain of reconstructive neurosurgery in 160 patients with traction and/or crush injury to the brachial plexus. In: Phantom and Stump Pain. Siegfried J and Zimmerman M (eds). 1st edition. Springer-Verlag, Berlin, pp126-147, 1981

16. Bruxelle J, Travers V and Thiebaut JB: Occurrence and treatment of pain after brachial plexus injury. Clin Orthop Relat Res 237: 87-95, 1988

17. von Schack D, Agostino MJ, Murray BS, Li Y, Reddy PS, Chen J, Choe SE, Strassle BW, Li C, Bates B, et al: Dynamic changes in the MicroRNA expression profile reveal multiple regulatory mechanisms in the spinal nerve ligation model of neuropathic pain. PLoS One 6: e17670, 2011

18. Choi Y, Yoon YW, Na HS, Kim SH and Chung JM: Behavioral signs of ongoing pain and cold allodynia in a rat model of neuropathic pain. Pain 59: 369-376, 1994.

19. Li X, Kroin JS, Kc R, Gibson G, Chen D, Corbett GT, Pahan K, Fayyaz S, Kim JS, van Wijnen AJ, et al: Altered spinal microRNA-146a and the microRNA-183 cluster contribute to osteoarthritic pain in knee joints. J Bone Miner Res 28 2512-2522, 2013

20. Wang L, Yuzhou L, Ying jie Z, Jie L and Xin Z: A new rat model of neuropathic pain: Complete brachial plexus avulsion. Neurosci Lett 589: 52-56, 2015.

21. Livak KJ and Schmittgen TD: Analysis of relative gene expression data using real-time quantitative PCR and the $2^{-\Delta \Delta C T}$ method. Methods 25: 402-408, 2001.

22. Ashburner M, Ball CA, Blake JA, Botstein D, Butler H Cherry JM, Davis AP, Dolinski K, Dwight SS, Eppig JT, et al; The Gene Ontology Consortium: Gene ontology: Tool for the unification of biology. Nat Genet 25: 25-29, 2000.

23. The Gene Ontology Consortium: Expansion of the Gene Ontology knowledgebase and resources. Nucleic Acids Res 45 D331-D338, 2017.

24. Kanehisa M and Goto S: KEGG: Kyoto Encyclopedia of Genes and Genomes. Nucleic Acids Res 28: 27-30, 2000.

25. Kanehisa M, Furumichi M, Tanabe M, Sato Y and Morishima K KEGG: new perspectives on genomes, pathways, diseases and drugs. Nucleic Acids Res 45: D353-D361, 2017.
26. Kanehisa M, Sato Y, Kawashima M,Furumichi M and Tanabe M: KEGG as a reference resource for gene and protein annotation. Nucleic Acids Res 44: D457-D462, 2016.

27. Zhang L, Zhang Y and Zhao ZQ: Anterior cingulate cortex contributes to the descending facilitatory modulation of pain via dorsal reticular nucleus. Eur J Neurosci 22: 1141-1148, 2005.

28. Wei F, Li P and Zhuo M: Loss of synaptic depression in mammalian anterior cingulate cortex after amputation. J Neurosci 19: 9346-9354, 1999

29. Porreca F, Ossipov MH and Gebhart GF: Chronic pain and medullary descending facilitation. Trends Neurosci 25: 319-325, 2002.

30. Robinson D, Calejesan AA and Zhuo M: Long-lasting changes in rostral ventral medulla neuronal activity after inflammation. J Pain 3: 292-300, 2002.

31. Vogt BA: Pain and emotion interactions in subregions of the cingulate gyrus. Nat Rev Neurosci 6: 533-544, 2005

32. Zhuo M: Cortical excitation and chronic pain. Trends Neurosci 31 199-207, 2008

33. Wei F and Zhuo M: Potentiation of sensory responses in the anterior cingulate cortex following digit amputation in the anaesthetised rat. J Physiol 532: 823-833, 2001.

34. Xu H, Wu LJ, Wang H, Zhang X, Vadakkan KI, Kim SS, Steenland HW and Zhuo M: Presynaptic and postsynaptic amplifications of neuropathic pain in the anterior cingulate cortex. $J$ Neurosci 28: 7445-7453, 2008.

35. Waggener CT, Dupree JL, Elgersma Y and Fuss B: CaMKII $\beta$ regulates oligodendrocyte maturation and CNS myelination. J Neurosci 33: 10453-10458, 2013

36. Fang M, Feng C, Zhao YX and Liu XY: Camk2b protects neurons from homocysteine-induced apoptosis with the involvement of HIF-1 $\alpha$ signal pathway. Int J Clin Exp Med 7: 1659-1668, 2014.

37. Miletic G, Hermes JL, Bosscher GL, Meier BM and Miletic V: Protein kinase $\mathrm{C}$ gamma-mediated phosphorylation of GluA1 in the postsynaptic density of spinal dorsal horn neurons accompanies neuropathic pain, and dephosphorylation by calcineurin is associated with prolonged analgesia. Pain 156: 2514-2520, 2015.

38. Yeh CY, Chung SC, Tseng FL, Tsai YC and Liu YC: Biphasic effects of chronic intrathecal gabapentin administration on the expression of protein kinase $\mathrm{C}$ gamma in the spinal cord of neuropathic pain rats. Acta Anaesthesiol Taiwan 49: 144-148, 2011.

39. Kim CH, Oh Y, Chung JM and Chung K: The changes in expression of three subtypes of TTX sensitive sodium channels in sensory neurons after spinal nerve ligation. Brain Res Mol Brain Res 95: 153-161, 2001.

40. Ma L, Uchida H, Nagai J, Inoue M, Chun J, Aoki J and Ueda H: Lysophosphatidic acid-3 receptor-mediated feed-forward production of lysophosphatidic acid: an initiator of nerve injuryinduced neuropathic pain. Mol Pain 5: 64, 2009.

41. Campbell JN and Meyer RA: Mechanisms of neuropathic pain. Neuron 52: 77-92, 2006

42. Nickel FT, Seifert F, Lanz S and Maihöfner C: Mechanisms of neuropathic pain. Eur Neuropsychopharmacol 2: 81-91, 2012.

43. Lu Y, Jian MY, Ouyang YB and Han RQ: Changes in rat brain microRNA expression profiles following sevoflurane and propofol anesthesia. Chin Med J (Engl) 128: 1510-1515, 2015

44. McAdams RM, McPherson RJ, Beyer RP, Bammler TK, Farin FM and Juul SE: Dose-dependent effects of morphine exposure on mRNA and microRNA (miR) expression in hippocampus of stressed neonatal mice. PLoS One 10: e0123047, 2015.

45. Gong Q, Lu Z, Huang Q, Ruan L, Chen J, Liang Y, Wang H, Yue $\mathrm{Y}$ and Feng S: Altered microRNAs expression profiling in mice with diabetic neuropathic pain. Biochem Biophys Res Commun 456: 615-620, 2015

46. McKiernan RC, Jimenez-Mateos EM, Sano T, Bray I, Stallings RL, Simon RP and Henshall DC: Expression profiling the microRNA response to epileptic preconditioning identifies miR-184 as a modulator of seizure-induced neuronal death. Exp Neurol 237: 346-354, 2012

47. Liu C, Teng ZQ, Santistevan NJ, Szulwach KE, Guo W, Jin P and Zhao X: Epigenetic regulation of miR-184 by MBD1 governs neural stem cell proliferation and differentiation. Cell Stem Cell 6: 433-444, 2010

This work is licensed under a Creative Commons

Attribution-NonCommercial-NoDerivatives 4.0 International (CC BY-NC-ND 4.0) License. 